Andreas Grau

Gewinnrealisierung nach International Accounting Standards 
GABLER EDITION WISSENSCHAFT 
Andreas Grau

\section{Gewinnrealisierung nach International Accounting Standards}

Mit einem Geleitwort von Prof. Dr. Roland Euler

Deutscher Universitäts-Verlag 
Die Deutsche Bibliothek - CIP-Einheitsaufnahme

Ein Titeldatensatz für diese Publikation ist bei

Der Deutschen Bibliothek erhältlich

Dissertation Universität Mainz, 2002

\section{Auflage September 2002}

Alle Rechte vorbehalten

(C) Deutscher Universitäts-Verlag GmbH, Wiesbaden, 2002

Lektorat: Brigitte Siegel / Nicole Schweitzer

Der Deutsche Universitäts-Verlag ist ein Unternehmen der Fachverlagsgruppe BertelsmannSpringer.

www.duv.de

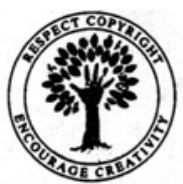

Das Werk einschließlich aller seiner Teile ist urheberrechtlich geschützt. Jede Verwertung außerhalb der engen Grenzen des Urheberrechtsgesetzes ist ohne Zustimmung des Verlags unzulässig und strafbar. Das gilt insbesondere für Vervielfältigungen, Übersetzungen, Mikroverfilmungen und die Einspeicherung und Verarbeitung in elektronischen Systemen.

Die Wiedergabe von Gebrauchsnamen, Handelsnamen, Warenbezeichnungen usw. in diesem Werk berechtigt auch ohne besondere Kennzeichnung nicht zu der Annahme, dass solche Namen im Sinne der Warenzeichen- und Markenschutz-Gesetzgebung als frei zu betrachten wären und daher von jedermann benutzt werden dürften.

Umschlaggestaltung: Regine Zimmer, Dipl.-Designerin, Frankfurt/Main

Gedruckt auf säurefreiem und chlorfrei gebleichtem Papier 


\section{Geleitwort}

Ein Kernproblem der externen Rechnungslegung ist der Zeitpunkt des Ausweises von Erträgen. Auf der Grundlage der International Accounting Standards (IAS) analysiert der Verfasser den Konflikt, der zwischen dem Ziel des Jahresabschlusses - Vermögensmehrungen möglichst vollständig darzustellen - und der Nebenbedingung der Nachprüfbarkeit - also nur hinreichend gesicherte Vermögensmehrungen zu zeigen besteht.

Der Verfasser weist überzeugend nach, daß - im Gegensatz zur objektivierungs- und vorsichtsgeprägten Umsatzgewinnkonzeption des Handelsgesetzbuches - die IAS einen wesentlich größeren Ermessensspielraum einräumen. Zwar erfolgt die ,income recognition“ auch auf einer ,accruals basis“, doch sind die Ansatzkriterien „probability of future economic benefit" und „reliability of measurement“ so unscharf, daß eine rechtssichere Beurteilung des Gewinnausweises kaum möglich ist.

Anhand ausgewählter Sachverhalte (Kaufverträgen mit Rücktritts- oder Rückgaberecht, Bill and hold sales, Lay away sales, Dividenden, langfristigen Auftragsfertigungen, Fremdwährungsgeschäften und Finanzinstrumenten) untersucht der Verfasser den genauen Zeitpunkt der Ertragsrealisation. Hierbei wird deutlich, daß den IAS operationalisierbare Realisationskriterien fehlen, so daß es dem Bilanzierenden im Einzelfall überlassen bleibt, ob er die Realisationskriterien als erfüllt ansehen will oder nicht.

Darüber hinaus belegt er überzeugend, daß den IAS ein einheitliches Konzept hinsichtlich der zugrundeliegenden Prinzipien der Ertragsrealisation fehlt. So werden im Regelfall - von rechtlichen Kategorien losgelöst - wirtschaftliche Leistungskriterien formuliert. In einigen Fällen (z.B. Dividendenerträgen) wird allerdings das Objektivierungserfordernis überbetont, indem lediglich formalrechtliche Kriterien ohne Rücksicht auf den wirtschaftlichen Gehalt vorgeschrieben werden. Dem Verfasser gelingt es damit, viele Illusionen hinsichtlich der Leistungsfähigkeit der internationalen Rechnungslegung zu zerstören. 


\section{Vorwort}

Der Zeitpunkt des Ertragsausweises stellt ein zentrales Bilanzproblem innerhalb der International Accounting Standards (IAS) dar. Die vorliegende Arbeit beabsichtigt auf Basis eines systematischen Vergleichs, die konzeptionellen Unterschiede zwischen den Ertragsrealisierungsgrundsätzen der IAS-Rechnungslegung und den handelsrechtlichen GoB herauszuarbeiten und die IAS-Realisationskriterien im Hinblick auf deren konkreten Beitrag zur Erreichung ihrer postulierten Zielsetzung zu untersuchen.

Mein ausdrücklicher Dank gilt an dieser Stelle meinem sehr geschätzten Doktorvater Herrn Professor Dr. Roland Euler für die Möglichkeit zur Promotion am Lehrstuhl für Allgemeine Betriebswirtschaftslehre und Betriebliche Steuerlehre, die Betreuung der Arbeit sowie seine jederzeitige Gesprächsbereitschaft. Herrn Prof. em. Dr. Joachim Knoth danke ich für die Übernahme des Zweitgutachtens.

Von ganzem Herzen danke ich meiner Frau für ihr Verständnis und ihre Unterstützung zur Verwirklichung meines Promotionsvorhabens.

Besonderen Dank schulde ich schließlich meinen Eltern. Ohne deren Fürsorge und Förderung meines gesamten bisherigen Lebensweges wäre die vorliegende Arbeit nicht denkbar. Ihnen sei diese Arbeit gewidmet.

Der Fachbereich Rechts- und Wirtschaftswissenschaften der Johannes GutenbergUniversität Mainz hat die vorliegende Arbeit, in leicht veränderter Form, unter dem Titel „Gewinnrealisierung nach International Accounting Standards - Zur Konzeption und Zieladäquanz der Gewinnrealisierungsgrundsätze des IASC“ im Jahre 2002 als Dissertation angenommen. 


\section{Inhaltsverzeichnis}

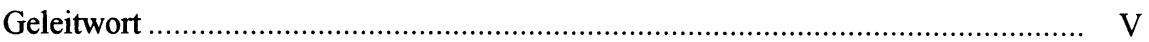

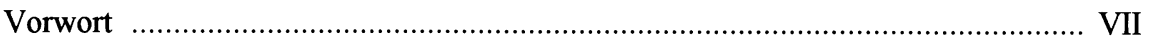

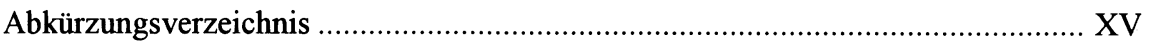

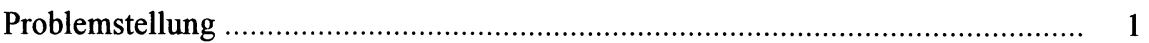

\section{Erstes Kapitel}

Bestimmungsgrundlagen der Rechnungslegung nach deutschem Handelsrecht und International Accounting Standards

A. Zur Bedeutung der handelsrechtlichen Grundsätze ordnungsmäßiger Buchführung.

I. Rechtsnormcharakter und Ermittlungsmethode der Grundsätze ordnungsmäßiger Buchführung.

II. Das Verständnis von den Grundsätzen ordnungsmäßiger Buchführung als einem offenen System ....................................................... 12

1. Der Systemgedanke.................................................................... 12

2. Die Struktur des inneren Systems ................................................. 14

III. Die ausschüttungsorientierte Primäraufgabe des handelsrechtlichen Jahresabschlusses

B. Vergleich mit den International Accounting Standards.................................. 21

I. Konzeptionelle Leitlinien ............................................................. 21

1. Elemente der IAS-Rechnungslegung ............................................... 21

2. Zur Adressatenkonkretisierung im framework ................................... 22 
3. Die Ziele und Funktionen des Jahresabschlusses nach IAS

a) Verdrängung des Prinzips der Gewinnanspruchsermittlung durch das Postulat der entscheidungsnützlichen Information über die finanzielle Lage und deren Veränderung sowie über die wirtschaftliche Leistung .......................................................... 24

aa) Informationsvermittlung zur Entscheidungsfindung................. 24

bb) Rechenschaft .................................................................. 26

b) Unterschiedliches Verständnis des Gewinnbegriffs ....................... 27

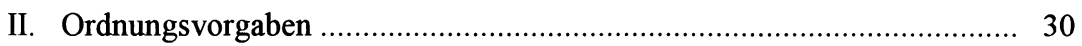

1. Normensetzungsproze $\beta$ und Rechtsnatur ......................................... 30

2. Das framework als deskriptive Basis der Zielvorstellungen des Jahresabschlusses nach International Accounting Standards ............... 36

a) Das framework .................................................................... 36

b) Stufenkonzeption ............................................................... 37

c) Frage nach dem inneren System ................................................ 38

\section{Zweites Kapitel}

Gewinnrealisation vor dem Hintergrund divergierender

Bestimmungsgrundlagen .....

A. Ausgangslage

I. Zur handelsrechtlichen Konzeption umsatzgebundener Gewinnermittlung

1. Das Realisationsprinzip als grundlegendes Prinzip handelsrechtlicher Rechnungslegung

a) Periodengerechte Gewinnermittlung nach Maßgabe des Realisationsprinzips

b) Anschaffungswertprinzip 45

2. Bestimmung des objektivierten Umsatzgewinns 48 
II. Vergleich mit den International Accounting Standards .......................... 52

1. Maßgeblicher Vorrang der Periodenabgrenzung .............................. 52

a) Konzeptionelle Grundlagen der accrual basis als Gewinnermittlungsnorm.

b) Die Bestimmung positiver Erfolgsbeiträge (income recognition)

aa) Die definitorischen Grundsätze des Begriffs „Ertrag“ (income)

bb) Die allgemeinen Ansatzkriterien (recognition criteria) 59

(1) Der Wahrscheinlichkeitsgrundsatz im Rahmen der income recognition

(2) Der Verläßlichkeitsgrundsatz im Rahmen der income recognition

2. Zur Bedeutung einer objektivierten Informationsvermittlung 64

a) Die Principal-Agent-Beziehung als Erklärungsansatz für die Objektivierungsnotwendigkeit im Rahmen der decision usefulness-Konzeption 64

b) Grundsätze der objektivierten Gewinnkonkretisierung nach IAS. 69

B. Konkretisierung des Erfolgsausweises 73

I. Die Konsequenzen des Ertragsrealisationsprinzips für den handelsrechtlichen Gewinnausweis 73

1. Konkretisierung des Realisationszeitpunktes 73

a) Das Prinzip des quasisicheren Anspruchs. 73

b) Bedeutung und Bedeutungsgrenzen des Zivilrechts im Rahmen der Gewinnrealisation 77

aa) Die regelmäßige Konkretisierung des Zeitpunktes des Gewinnausweises durch den Übergang der Preisgefahr 
bb) Der Rückgriff auf die typisierende Beurteilung des quasisicheren Gewinns durch den Erwerb des wirtschaftlichen Eigentums

2. Anschaffungskosten umsatzinduzierter Forderungen ......................... 82

II. Vergleich mit den International Accounting Standards .......................... 84

1. Analyse der konzeptionellen Voraussetzungen zur Bestimmung des Zeitpunktes der income recognition................................................ 84

2. Konkretisierung der Realisierungszeitpunkte ……............................. 88

a) Realisation im engeren Sinne .................................................... 88

aa) Die Konkretisierung des Wahrscheinlichkeitsgrundsatzes (probability of future economic benefits) ……......................... 88

(1) Übertragung der maßgeblichen mit dem Eigentum verbundenen Risiken und Chancen ................................... 88

(2) Wegfall des fortdauernden Verfügungsrechts und der wirksamen Kontrolle

(3) Wahrscheinlichkeit des Zuflusses wirtschaftlichen

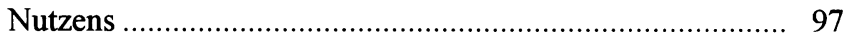

(4) Ausgewählte Einzelfälle ................................................. 98

(a) Kaufvertrag mit Rücktritts- oder Rückgaberecht .......... 98

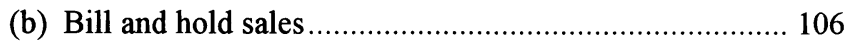

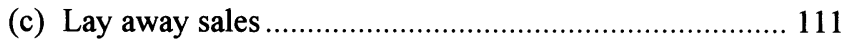

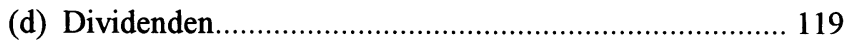

bb) Die Konkretisierung des Verläßlichkeitsgrundsatzes (reliability of measurement)

(1) Das Erfordernis der zuverlässigen Ertrags- und Aufwandsbemessung

(2) Zur Ertragsbemessung unter Berücksichtigung des Zinsaspektes

(3) Gewinnrealisierung bei Tauschgeschäften 
b) Der Erfolgsausweis bei langfristiger Auftragsfertigung ................... 140

aa) Voraussetzungen der percentage of completion-Methode .......... 140

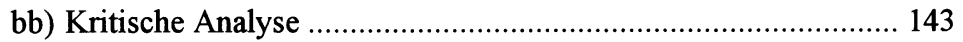

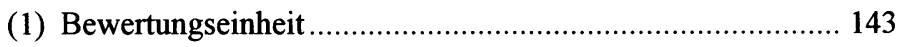

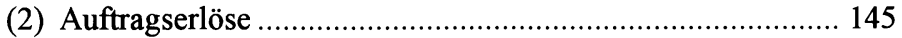

(3) Auftragskosten ............................................................... 150

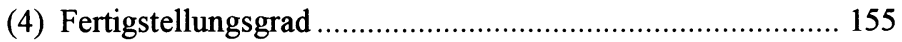

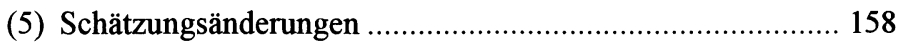

(6) Antizipation erwarteter Verluste ........................................ 159

cc) Würdigung vor dem Hintergrund einer zweckgerichteten

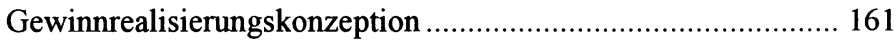

c) Umrechnung von Fremdwährungsgeschäften ................................ 171

d) Bewertung und Realisation bei ausgewählten

Finanzinstrumenten

Zusammenfassende Thesen

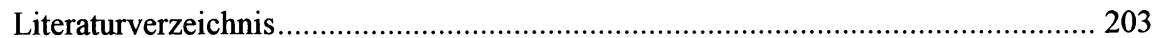

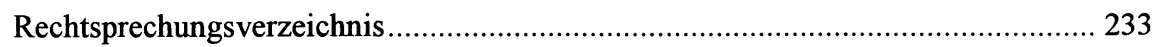

Verzeichnis der Gesetze und Gesetzesmaterialien ................................................... 235 


\section{Abkürzungsverzeichnis}

a.a.O. am angegebenen Ort

Abs.

Absatz

Abt.

Abteilung

AG

Die Aktiengesellschaft (Zeitschrift)

AICPA

American Institute of Certified Public Accountants

AktG

Aktiengesetz

Anm.

Anmerkung

ARB

Accounting Research Bulletin

ASR

Accounting Series Releases

Aufl.

Auflage

BB

Betriebs-Berater (Zeitschrift)

$\mathrm{Bd}$.

Band

bearb.

bearbeitet

$\mathrm{BFH}$

Bundesfinanzhof

BFHE

Sammlung der Entscheidungen des Bundesfinanzhofs

BFuP

Betriebswirtschaftliche Forschung und Praxis (Zeitschrift)

BGB

Bürgerliches Gesetzbuch

BGB1

Bundesgesetzblatt

BGHZ

Sammlung der Entscheidungen des Bundesgerichtshofs in Zivilsachen

bspw. beispielsweise

BStBl Bundessteuerblatt

BVerfGE Sammlung der Entscheidungen des Bundesverfassungsgerichts

DB

Der Betrieb (Zeitschrift)

DRSC

Deutsches Rechnungslegungs Standards Committee e.V.

DStR

Deutsches Steuerrecht (Zeitschrift)

DStZ

Deutsche Steuerzeitschrift (Zeitschrift) 
EFG Sammlung der Entscheidungen der Finanzgerichte

EGHGB Einführungsgesetz zum Handelsgesetzbuch

EStG Einkommensteuergesetz

EU Europäische Union

EuGH Europäischer Gerichtshof

EuGH-Slg. Sammlung der Rechtsprechung des Europäischen Gerichtshofs

FASB Financial Accounting Standards Board

ff. und die folgenden (Paragraphen, Seiten)

FG Finanzgericht

FN-IDW Fachnachrichten des Instituts der Wirtschaftsprüfer in Deutschland

e.V.

FR Finanzrundschau (Zeitschrift)

GEFIU Gesellschaft für Finanzwirtschaft in der Unternehmensführung e.V.

GoB Grundsätze ordnungsmäßiger Buchführung

GrS Großer Senat

HGB Handelsgesetzbuch

hrsg. herausgegeben

Hrsg. Herausgeber

Hs. Halbsatz

i.V.m. in Verbindung mit

IAS International Accounting Standards

IASC International Accounting Standards Committee

IDW Institut der Wirtschaftsprüfer in Deutschland e.V.

insb. insbesondere 
JbDStJG Jahrbuch der Deutschen Steuerjuristischen Gesellschaft

JbFfSt Jahrbuch der Fachanwälte für Steuerrecht

Jg. Jahrgang

krp Kostenrechnungspraxis (Zeitschrift)

m.w.N. mit weiteren Nachweisen

NB Neue Betriebswirtschaft (Zeitschrift)

No. Number

Nr. Nummer

OFD Oberfinanzdirektion

RFHE Sammlung der Entscheidungen des Reichsfinanzhofs

RIW Recht der Internationalen Wirtschaft (Zeitschrift)

Rn. Randnummer(n)

RStBl Reichssteuerblatt

S. Seite

SAB Staff Accounting Bulletin

SEC Securities and Exchange Commission

SFAC Statement of Financial Accounting Concepts

SFAS Statement of Financial Accounting Standards

SIC Standing Interpretations Committee

SOP Statement of Position

Sp. Spalte

StbJb Steuerberater-Jahrbuch

StbKongR Steuerberater-Kongreß-Report

StuW Steuer und Wirtschaft (Zeitschrift) 
XVIII

$\mathrm{StVj}$ Steuerliche Vierteljahresschrift

Tz. Textziffer

u. und

u.a. und andere, unter anderem

USA United States of America

US-GAAP United States Generally Accepted Accounting Principles

Verf. Verfasser

vgl. vergleiche

Vol. Volume

WPg Die Wirtschaftsprüfung (Zeitschrift)

z.B. zum Beispiel

ZfB Zeitschrift für Betriebswirtschaft

$\mathrm{ZfbF} \quad$ Zeitschrift für betriebswirtschaftliche Forschung

ZfhF Zeitschrift für handelswissenschaftliche Forschung

ZGR Zeitschrift für Unternehmens- und Gesellschaftsrecht 